\title{
SIMULAÇÃO COMPUTACIONAL APLICADA À ANÁLISE OPERACIONAL DE SERVIÇO FEEDER PARA A CABOTAGEM DO ESTADO DO AMAZONAS
}

\author{
Rafael Lima Medeiros \\ Instituto de Pesquisa em Transporte. Av. Ferreira Pena, 1144 - Centro. Manaus - AM. \\ rafa.comp_adm@hotmail.com \\ José Teixeira de Araujo Neto Santos \\ Instituto de Pesquisa em Transporte. Av. Ferreira Pena, 1144 - Centro. Manaus - AM. \\ teixeira_amb@hotmail.com \\ Poliana Cardoso \\ Instituto de Pesquisa em Transporte. Av. Ferreira Pena, 1144 - Centro. Manaus - AM. \\ teixeira_amb@hotmail.com
}

\section{Nelson Kuwahara}

Universidade Federal do Amazonas. Av. Rodrigo Octavio, 3000 - Coroado. Manaus - AM. kuwaharanelson@yahoo.com.br

\section{Waltair Vieira Machado}

Universidade Federal do Amazonas. Av. Rodrigo Octavio, 3000 - Coroado. Manaus - AM. waltair_machado@yahoo.com.br

\section{Resumo}

No estado do Amazonas a cabotagem é utilizada principalmente para transportar carga geral e contêineres, sendo que a movimentação de carga conteinizada possui o maior potencial de crescimento devido a existência do Polo Industrial de Manaus (PIM). Neste trabalho é proposto modelos de simulação computacional para analisar o comportamento operacional da cabotagem do estado do Amazonas utilizando serviço feeder como alternativa. Os resultados apontaram que a viabilidade operacional do uso de serviço feeder depende do equilíbrio entre a demanda por movimentação de contêineres e a capacidade de movimentação do porto concentrador, sendo este necessário para operar com serviços feeders. Portanto, o estudo revela que é necessário equacionar o volume de cargas conteinizadas que o estado é capaz de gerar e atrair, para evitar que ocorra investimentos ineficientes na criação de infraestrutura de transporte que não atendam a adequadamente a demanda real ou estimada.

Palavras-chave: Cabotagem. Simulação. Transporte aquaviário.

\begin{abstract}
In the state of Amazonas cabotage is mainly used to transport general cargo and containers but cargo handling conteinizada has the highest growth potential due to the existence of the Industrial Pole of Manaus (PIM). This paper proposes computer simulation models to analyze the operational behavior of cabotage state of Amazonas using feeder service as an alternative. The results showed that the operational viability of the use of feeder service depends on the
\end{abstract}


balance between the demand for container handling and the handling capacity of the port concentrator, which is required to operate feeder services. Therefore, the study shows that it is necessary to equate the volume of cargo in container that the state is able to generate and attract to help prevent inefficient investments in creating transportation infrastructure that does not adequately meet the actual demand or estimated.

Keywords: cabotage. simulation. water transport

\section{INTRODUÇÃO}

A cidade de Manaus, principal polo de atração de viagens do estado do Amazonas, é um caso típico de localidade com múltiplas alternativas para o escoamento de sua produção, basicamente industrial. No entanto, variáveis ambientais e estruturais limitam as opções de escolha dos armadores, aumentando assim os custos e o transit time das operações de movimentação de cargas.

A principal forma de escoamento de cargas do estado do Amazonas é o uso conjunto dos modais rodoviário e fluvial, ou seja, as cargas com origem Manaus são transportadas até um porto com acesso rodoviário as demais regiões do país.

Este processo é ineficiente em muitos casos, pelos altos custos operacionais, baixa taxa de ocupação de barcos e caminhões e pela baixa confiabilidade nos tempos de entrega [6]. Neste contexto, o transporte de cabotagem é uma alternativa viável para atender a demanda de serviços de transporte de cargas com maior nível de competividade para a região.

Contudo, o atual estágio de desenvolvimento do transporte de cabotagem no Amazonas não permite que este modal absorva totalmente a demanda potencial existente para o mercado regional. Sendo os principais entraves para o desenvolvimento do transporte de cabotagem no Amazonas relacionados com a infraestrutura física e operacional portuária, inadequada para atender grandes escalas de demanda por este serviço [9].

Neste trabalho é proposto modelos de simulação computacional para analisar o comportamento operacional da cabotagem do estado do Amazonas utilizando serviço feeder como alternativa.

\section{SERVIÇO FEEDER}

Em países como EUA e alguns da União Europeia, a cabotagem encontra-se em um estágio de desenvolvimento superior aos encontrados no Brasil. Basicamente, tal fato deve-se às diferenças no nível de desenvolvimento das infraestruturas portuárias, nível de integração entre os entes que compõe o sistema de transporte aquaviário e as tecnologias empregadas na gestão e operação do sistema nesses países [10].

Nos países onde a cabotagem é mais desenvolvida são utilizados serviços feeders (alimentadores) para a obtenção de cargas em portos com possibilidade de atracação de navios de grande porte, normalmente portos concentradores de cargas ( $h u b$ ports), racionalizando o fluxo de cargas, normalmente conteinizada [5].

Serviço feeder é o transporte que ocorre na modalidade porto-a-porto, tanto para contêineres vazios quanto cheios, sendo especialmente importante para armadores de carga de navegação longo curso [7].

Na definição de [3], o serviço feeder é constítuido por operações de movimentação de grandes volumes de cargas de importação e de exportação, em portos concentradores, para posterior distribuição para portos e terminais portuários de menor capacidade, em navios de menor porte (feeders), na navegação de cabotagem. Os navios feeders transportam, em média, 
entre 500 e 1100 TEU.

\subsection{REDES HUB-AND-SPOKE}

A lógica de funcionamento de uma rede de transporte que utiliza pontos concentradores (hubs) e pontos alimentadores (feeders) normalmente é representada por redes do tipo "hub-and-spoke". Ou seja, existem nós específicos para a concentração de cargas $(h u b)$, que formam os corredores principais para o escoamento de cargas, por outro lado, existem nós menores (spokes) com influência importante apenas em contexto regional.

Estes nós menores são atendidos e alimentam um ou mais dos nós concentradores através de serviços feeders. Um caso prático de como funciona um sistema "hub-and-spoke" é discutido no exemplo de [13] apresentado na Figura, 1 a seguir:

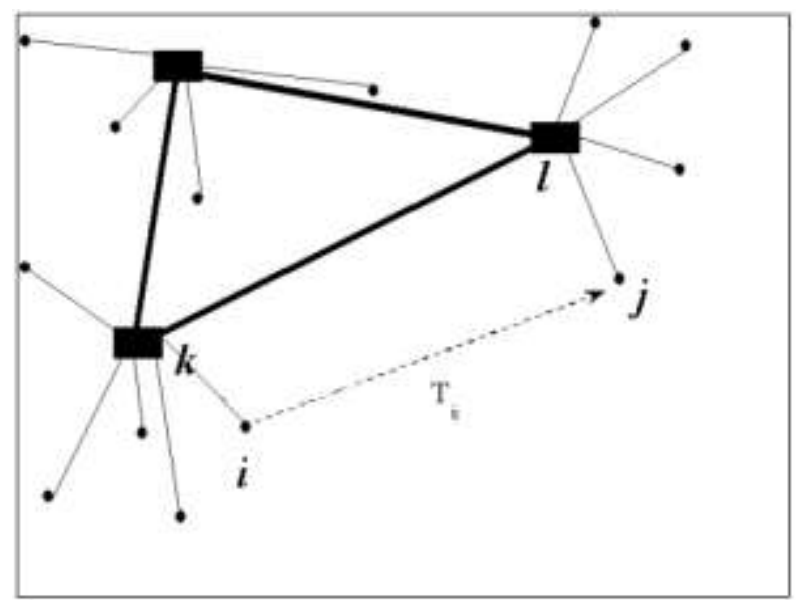

Figura 1: Exemplo de rede do tipo hub-and-spoke Fonte: Silva e Cunha [13].

Os nós $k$ e $l$ representam os terminais para consolidação de carga ("hubs") e os nós $i$ e $j$ aqueles que são atendidos através dos terminais ("spokes"). Um fluxo de carga $\mathrm{T}_{i j}$, com origem do nó $i$ e destino $j$, é atendido da seguinte forma: inicialmente a carga em $i$ é enviada para o terminal de consolidação (" $h u b ") ~ k$, onde é consolidada com outras cargas e enviada para o " $h u b " ~ l$ e daí distribuída para os pontos atendidos por $l$, incluindo o nó $j$ [13].

\section{CENÁRIOS LOGÍSTICOS ALTERNATIVOS}

O transporte de cabotagem no Amazonas apresenta indicadores significativos frente à movimentação nacional de cargas por meio deste tipo de navegação, com cerca de $15,46 \%$ do volume total transportado em 2010.

A cidade de Manaus é a única localização considerada como porto de origem, devido suas características econômicas, e por possui um importante polo industrial, que gera e atrai praticamente toda a demanda por movimentação de contêineres, bem como pelo fato de os terminais privados, que movimentam estes tipos de carga no estado, estarem localizados na cidade.

\subsection{CEnÁRIO REAL}

O município de Manaus possui dois Terminais de Uso Misto Privativo (TUP) para embarque e desembarque de carga de contêineres. Ambos estão situados na margem esquerda do rio Negro, possuem píer flutuante conectados por uma ponte de acesso com cais de atracação, oferecendo duas posições de acostagem, berços internos e externos [12].

Segundo [11] são 58 pontos de atracação situados na orla do Rio Negro nas 
circunvizinhanças de Manaus, sendo $21 \%$ terminais autorizados. Quanto aos serviços prestados, 10 (dez) foram classificados como estaleiros, 48 (quarenta e oito) terminais privados, sendo 1 (um) serviço offshore e 8 (oito) são empresas de navegação.

Manaus atua como porto concentrador regional de cargas, desta forma o uso do modal marítimo, seja na navegação de longo curso ou na cabotagem, é realizado diretamente com o porto de destino.

As empresas localizadas na cidade para enviar ou receber contêineres realizam os processos de carregamento ou descarregamento em algum TUP da cidade, que por sua vez movimenta esta carga via transporte marítimo diretamente com o porto final ou de origem. No porto de destino a carga segue para o processo de distribuição até o cliente final, normalmente utilizando outros tipos de modais como rodovias ou ferrovias. Destaca-se uma rota importante para o escoamento da produção do estado. Utiliza-se do modal fluvial até Belém para chegar com cargas até São Paulo, via modal rodoviário, conforme a Figura 2.

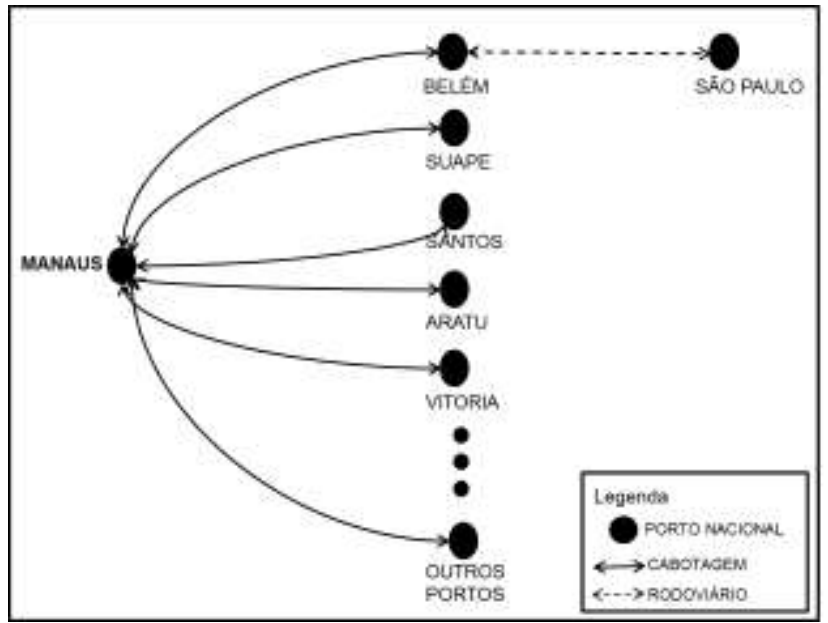

Figura 2: Modelagem do transporte de cabotagem do estado do Amazonas.

Em relação à movimentação de contêineres, nota-se na análise dos principais fluxos a partir de Manaus que a sua área de influência está situada no contexto nacional e internacional. As maiores influências de Manaus estão principalmente nos portos de Coari, Aratu, Santos, São Sebastião, Rio de Janeiro entre outros [12].

\subsection{CenÁrios Alternativos}

Os cenários alternativos significam a proposta de uma nova configuração dos fluxos de carga conteinizada a partir da adoção de um porto concentrador na região de Curuça nas proximidades de Belém. Nestes cenários os contêineres com origem ou destino Amazonas realizam o trajeto Manaus-porto concentrador via embarcações tipo feeder. No porto concentrador são transbordadas para embarcações maiores para realizar outro trajeto maior para diversos portos finais via cabotagem, longo curso ou outro tipo de modal.

A Figura 3 ilustra como tais cenários representariam uma mudança nos fluxos de cargas, com a existência de um porto concentrador, servindo de acesso à cidade Manaus e aos demais portos regionais menores. Note-se, ainda, que este superterminal influenciaria todos os estados da Amazônia Ocidental. 


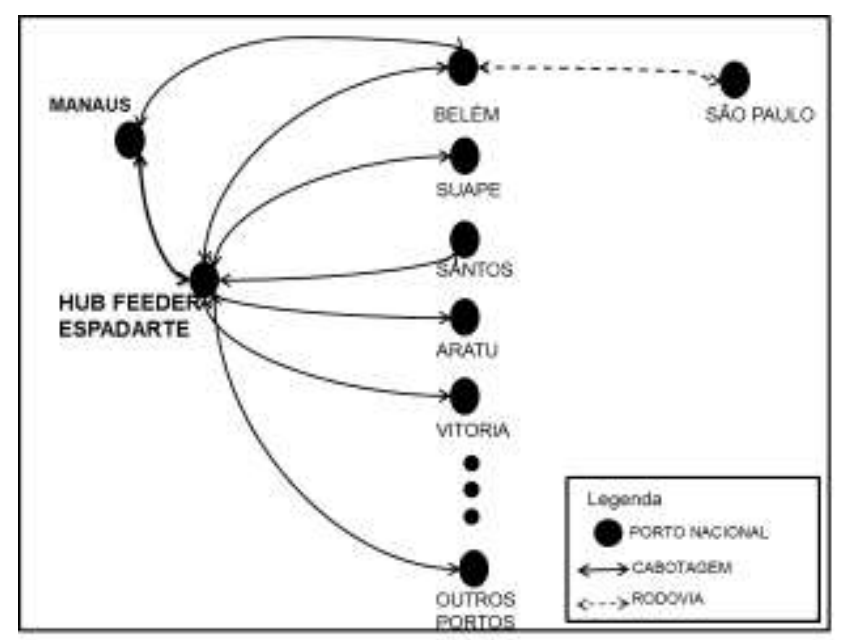

Figura 3: Modelagem do transporte de cabotagem do Amazonas com um porto concentrador.

Os cenários a serem simulados visam avaliar quais os níveis de demanda são suportados pela rede hub-and-spoke. Outro objetivo relevante é determinar quais as capacidades de movimentação do porto concentrador são mais adequadas para atender os diferentes níveis de demanda que Manaus pode vir a atrair com a modernização do sistema de cabotagem atual. A intersecção dessas duas variáveis permite gerar os cenários apresentados no Quadro 1 a seguir:

Quadro 1: Cenários alternativos para a cabotagem do estado do Amazonas operando com serviço feeder

\begin{tabular}{|l|l|l|l|}
\hline \multicolumn{1}{|c|}{ Variáveis } & \multicolumn{3}{c|}{ Capacidade do porto concentrador } \\
\hline Níveis de Demanda & Regional & Nacional & Internacional \\
\hline Demanda Prevista (2015) & Cenário 1 & Cenário 2 & Cenário 3 \\
\hline Demanda Potencial & Cenário 4 & Cenário 5 & Cenário 6 \\
\hline Super Demanda & Cenário 7 & Cenário 8 & Cenário 9 \\
\hline
\end{tabular}

O ganho operacional esperado com uso dessa abordagem está na redução no tempo de processamento das embarcações menores e no aumento da velocidade do fluxo de cargas com o fracionamento do envio dos contêineres.

\section{MODELOS E SIMULAÇÃO COMPUTACIONAL}

Para fins de modelagem, a cidade de Manaus foi tomada como único porto representativo para a cabotagem no estado, devido a grande concentração que a cidade exerce sobre a entrada e saída de carga para a Região, conforme já discutido anteriormente. No entanto, a distribuição espacial dos principais fluxos de contêineres por cabotagem coloca Manaus como o porto mais afastado dentre aqueles que fazem parte dos principais movimentadores de carga conteinizada.

\subsection{MODELO DE TRANSPORTE DE CABOTAGEM REAL}

O objetivo do modelo do transporte de cabotagem real é conseguir determinar quanto tempo os TUP da cidade de Manaus consomem para realizar todas as operações portuárias necessárias para operacionalizar o fluxo de carga entre a chegada e a saída das embarcações, permitindo reproduzir o comportamento do sistema real. O diagrama dos processos de funcionamento de um terminal portuário movimentador de contêineres utilizado neste 
trabalho é mostrado na Figura 4. As macro-etapas do processo de movimentação de carga que representam a movimentação dos TUP locais são assim descritas:

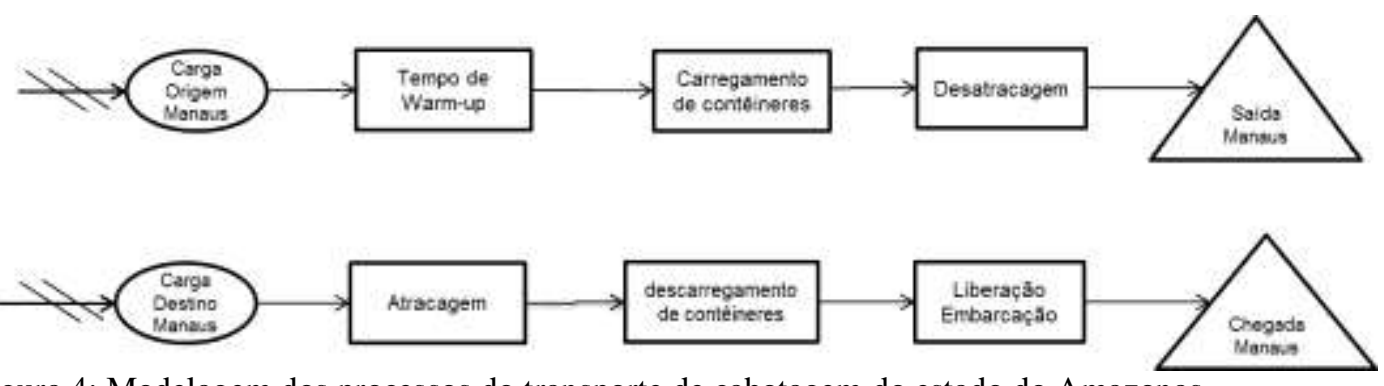

Figura 4: Modelagem dos processos do transporte de cabotagem do estado do Amazonas.

A partir das etapas de funcionamento de um terminal portuário de contêineres, apresentado na Figura 4, foi possível definir um modelo representativo do sistema real, inserindo os recursos utilizados, o compartilhamento dos recursos e o ciclo de informação que permeiam os processos portuários. Esta modelagem é apresentada na Figura 5:

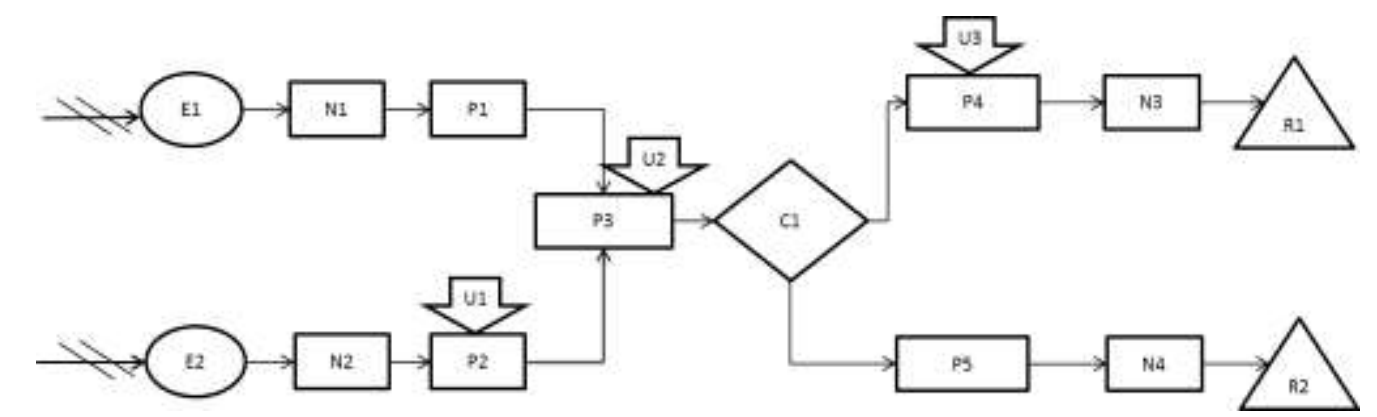

Figura 5: Modelo de simulação do transporte de cabotagem real do estado do Amazonas.

Os blocos da Figura 5 são relativos aos processos e recursos utilizados. Já os valores para os parâmetros foram obtidos junto a ANTAQ [2], através da amostragem de 32 valores referentes aos meses entre janeiro de 2010 a agosto de 2012 relativos ao desempenho operacional dos TUP de Manaus, presentes no sistema de acompanhamento de desempenho portuário da ANTAQ. Já os valores para as variáveis de chegada e saída de carga conteinizada para o estado do Amazonas foram obtidos junto ao Sistema de Informação da Navegação Interior [14] (SINI), contemplando o período de janeiro a novembro de 2012.

Os valores coletados junto a ANTAQ constituíram uma série para cada variável operacional, desta forma utilizando o software Input Analyzer foi possível obter a melhor distribuição estatística que explica a movimentação portuária no período estudado. Já os dados de custos médios totais foram obtidos diretamente com a ANTAQ [1]

Os dados de entradas para o modelo são: tempos de operações; distribuições de chegadas de cargas; custo por unidade de carga; e recursos portuários. Já os dados de saídas são: tempo total de operação; fila; tempo de espera; custo total; quantidade total movimentada.

\subsection{MODELOS ALTERNATIVOS DE TRANSPORTE DE CABOTAGEM}

A Figura 6, a seguir, apresenta os principais processos do funcionamento dos TUP de Manaus e do porto concentrador operando em conjunto. Esta configuração está baseada no conceito de rede hub-and-spoke. 


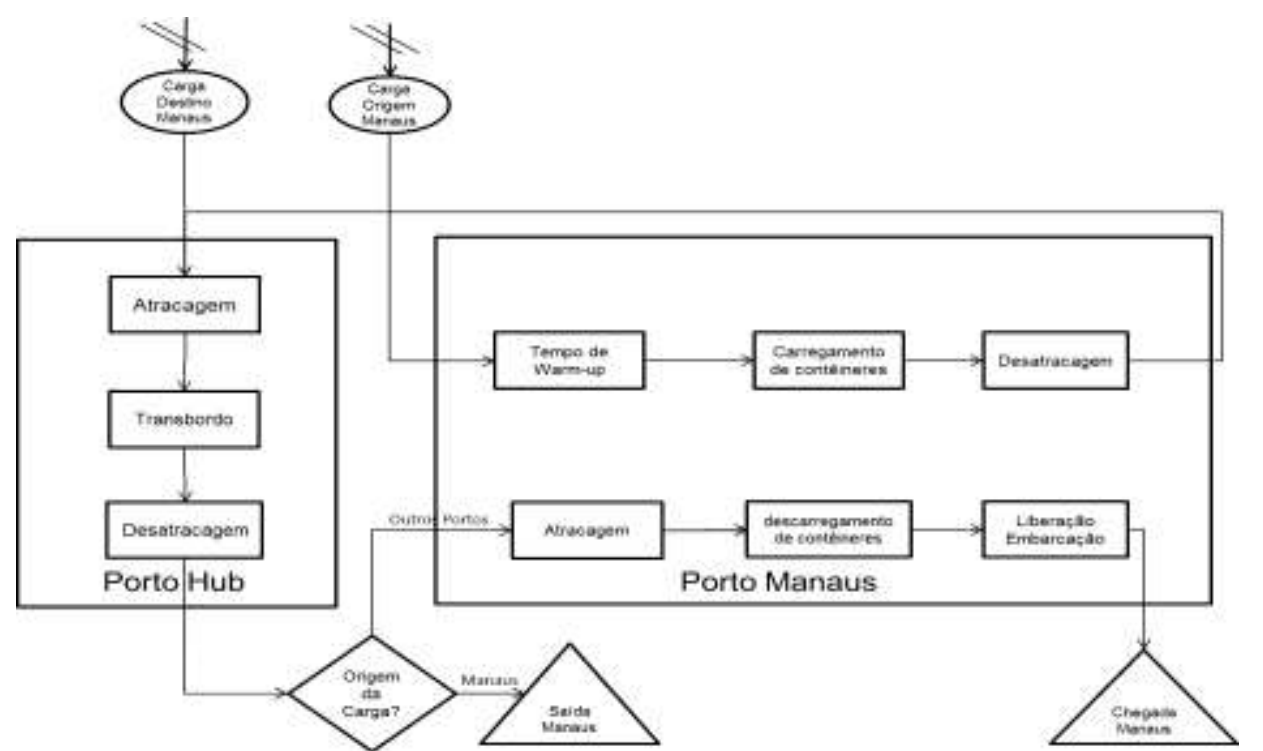

Figura 6: Modelagem dos processos do transporte de cabotagem operando com serviço feeder

A Figura 6 evidencia que a quantidade de processos em uma rede hub-and-spoke aumenta consideravelmente em relação ao cenário real com a criação de um porto concentrador operando integrado aos TUP de Manaus.

A Figura 7, a seguir, apresenta a modelagem dos processos da Figura 6 para fins de simulação do sistema operando em uma rede hub-and-spoke, de forma similar a modelagem do transporte real. É relevante notar que os processos referentes aos TUP de Manaus não são alterados, as mudanças realizadas são a diminuição do tempo de operação, por necessitar de menos tempo para movimentar uma quantidade de carga menor em embarcações feeder, e a maior frequência de chegada de embarcações, por se tratar de navios feeder.

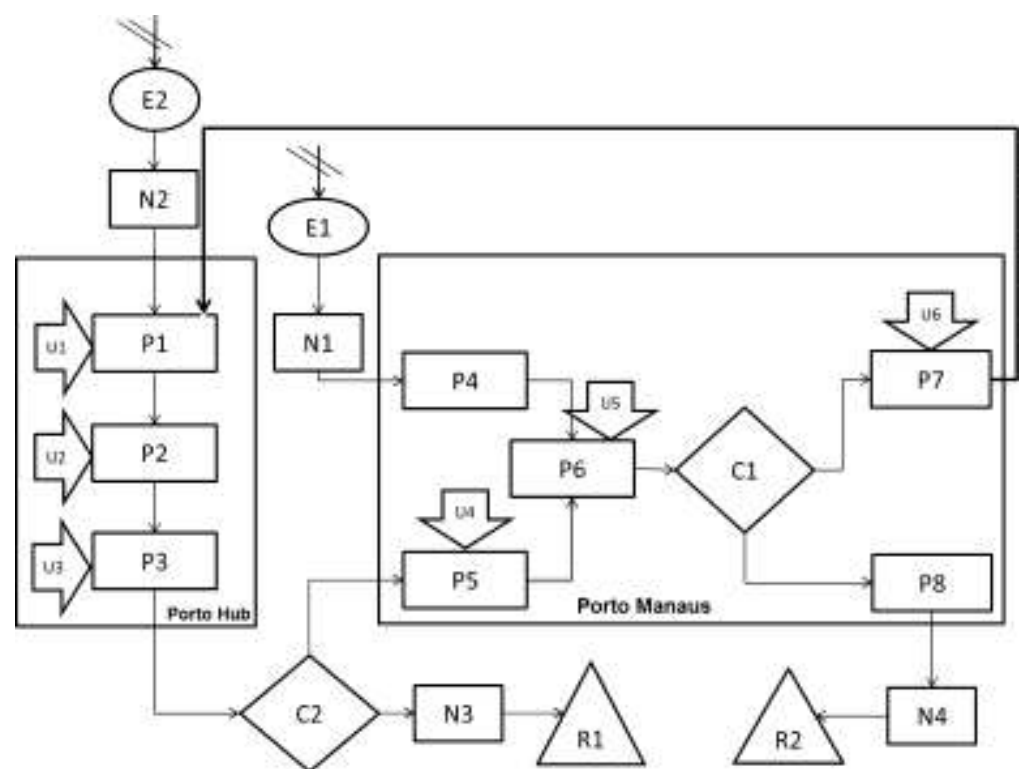

Figura 7: Modelo de simulação do transporte de cabotagem operando com serviço feeder

Os dados de entradas para o modelo, tanto para os TUP de manaus quanto para o porto concentrador, são: tempos de operações; distribuições de chegadas de cargas; custo por unidade de carga; e recursos portuários. Já os dados de saídas são: tempo total de operação; fila; tempo de espera; custo total; quantidade total movimentada. 


\subsection{AMBIENTE DE SIMULAÇÃo ARENA}

O ARENA ${ }^{\circledR}$ é um software de simulação lançado em 1993 pela empresa System Modeling, tendo sido apresentado como sucessor de outras aplicações de simulação mais antigas como o SIMAN e o CINEMA. A System Modeling foi posteriormente incorporada pela Rockwell Software, atual distribuidora do ARENA [8].

O software escolhido para realizar a simulação apresentada é o ARENA ${ }^{\circledR}$ Rockwell Software Inc. $11.0^{\circledR}$ versão acadêmica proprietária, disponilizada para institutos de pesquisas e/ou ensino que desenvolvam estudos no campo da simulação computacional. Já a metodologia utilizada para o desenvolvimento do projeto do modelo de simulação foi a de [4].

A escolha do ARENA deve-se ao fato deste ser um simulador genérico, sendo flexível para representar processos produtivos, comportamento de redes de transporte e decisões lógicas que precisam ser consideradas durante o funcionamento de um terminal portuário movimentador de contêiner inserido em um contexto de malha aquaviária.

\section{ANÁLISE E DISCUSSÃO DE RESULTADOS}

As simulações foram realizadas com 100 replicações para um tempo de operação de 334 dias seguidos, ou seja, onze meses de operação, para a modelagem do transporte de cabotagem real. E 100 interações de 365 dias (1 ano) para a simulação dos cenários alternativos.

\subsection{RESULTADOS DA SIMULAÇÃO DO MODELO DE TRANSPORTE CABOTAGEM REAL}

A Figura 8 apresenta o comportamento do volume de cargas movimentadas ao longo do ano de 2012 em comparação com o resultado obtido com a simulação. O volume de carga movimentado pelo transporte de cabotagem no ano de 2012 é descrito pela curva "Total Real Manaus", enquanto que o resultado da simulação é denominado "Total Simulado Manaus".

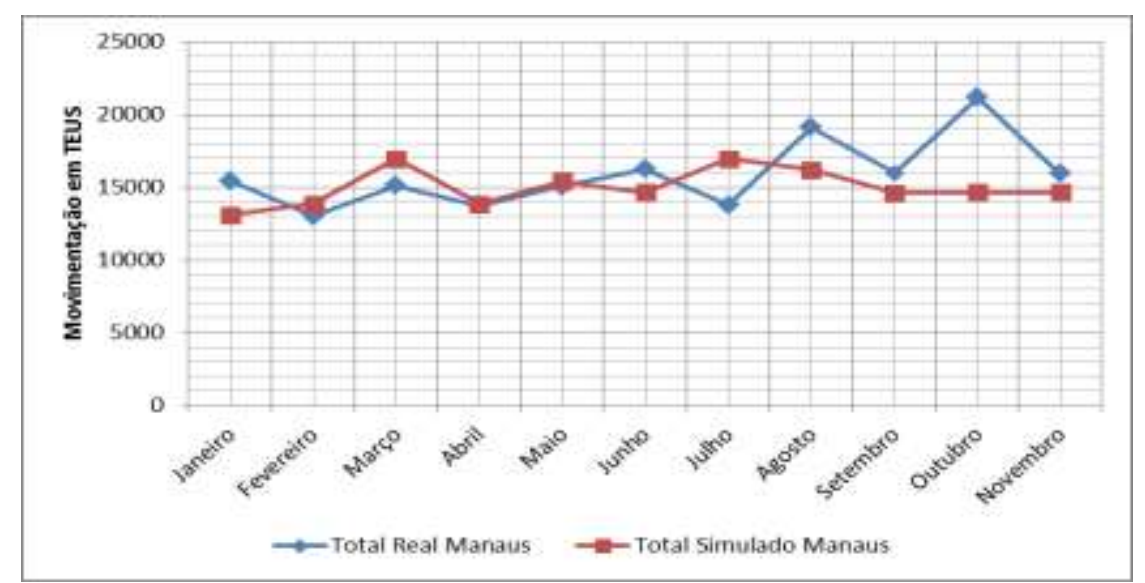

Figura 8: Quantidade de cargas movimentadas em 2012 versus a quantidade de cargas movimentadas na simulação.

A Figura 8 permite inferir que a simulação obtem boa proximidade com os dados reais, a exceção do mês último trimestre devido a sazonalidade do mercado. O resumo dos resultados do desempenho operacional dos TUP de Manaus obtidos na simulação do transporte real de cabotagem é apresentado no Quadro 2. As informações de "Tempo Médio de Espera" e a "Quantidade Média na Fila de Operação Portuária" não são informações estatísticas presentes nos bancos de dados pesquisados, por este motivo não é possível fazer comparação direta com o transporte de cabotagem real nesses itens. 
Quadro 2: Comparação dos dados do transporte de cabotagem real e os resultados da simulação

\begin{tabular}{|l|r|r|r|}
\hline Respostas & $\begin{array}{l}\text { Transporte cabotagem } \\
\text { real }\end{array}$ & $\begin{array}{l}\text { Simulação } \\
\text { real }\end{array}$ & cabotagem \\
\hline Tempo médio de operação (h) & 36,90 & 36,87 \\
\hline $\begin{array}{l}\text { Quantidade média na fila de } \\
\text { operação portuária (navios) }\end{array}$ & - & 1,50 \\
\hline Tempo médio de espera (h) & - & 93,20 \\
\hline Custo total de movimentação (R\$) & $29.594 .613,00$ & $27.689 .162,58$ \\
\hline Quantidade movimentada (TEU) & 174.608 & 164.882 \\
\hline Tempo médio total de operação (h) & - & 139,42 \\
\hline Tempo médio de atracagem (h) & 1,20 & 1,30 \\
\hline Tempo médio de desatracagem (h) & 6,27 & 6,80 \\
\hline Tempo médio de warm-up (h) & 2,90 & 2,80 \\
\hline $\begin{array}{l}\text { Tempo médio de liberação da } \\
\text { embarcação (h) }\end{array}$ & 7,47 & 7,60 \\
\hline
\end{tabular}

Os testes realizados para validar o modelo de simulação do transporte de cabotagem real apresentaram resultados satisfatórios, pois no primeiro momento, quando buscou-se verificar se os resultados operacionais simulados apresentavam boa proximidade com os dados reais, foi constatado que todos os indicadores operacionais simulados apresentavam variação menor que $6 \%$ em relação aos dados reais. Sendo as diferenças de custo e quantidade movimentada serem explicadas basicamente pela quantidade de carga em processamento, que por questões de modelagem, apenas são computadas como resultado quando passam por todas as etapas do modelo.

\subsection{RESUltados DA SimulaÇÃo dOS MODElos ALTERNATIVOS DE TRANSPORTE CABOTAGEM}

Os cenários 4 e 7 não puderam ser concluídos devido o grande desbalanceamento entre capacidade operacional e demanda por movimentação de cargas, que gera uma quantidade grande de entidades dentro do modelo, simultaneamente ao crescimento exponencial de embarcações em fila. Desta forma, é possível verificar, de forma preliminar à conclusão das respectivas simulações, que estes cenários são muito ineficientes em relação aos demais.

Os principais resultados dos demais cenários alternativos executados são: Taxa de ocupação, Tempo total médio de operação e Tempo total médio de espera. A Figura 9, a seguir, ilustra o comportamento da variável taxa de ocupação das operações portuárias de carregamento e descarregamento.

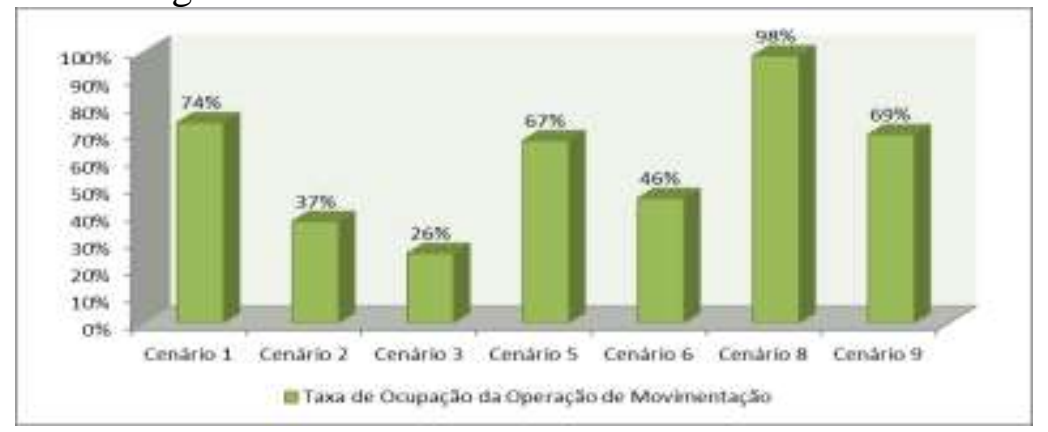

Figura 9: Taxa de ocupação das operações de movimentação 
Os Cenários 2, 3 e 6 apresentam baixa taxa de ocupação, da ordem de 37\%, 26\% e $46 \%$ respectivamente. Condição inversa ao Cenário 8 que opera de forma saturada, com taxa de ocupação de $98 \%$, próximo do limite da capacidade de movimentação. Já os Cenários 1, 5 e 9 apresentam taxa de ocupação em torno de $70 \%$ o que permite opera sem gerar maiores gargalos e com possibilidade de aumento do volume transportável.

O tempo total médio de operação expressa quanto tempo a carga consome desde a entrada até a saída do sistema, considerando tempos de processamento e os tempos em espera. Os resultados deste indicador estão apresentados na Figura 10:

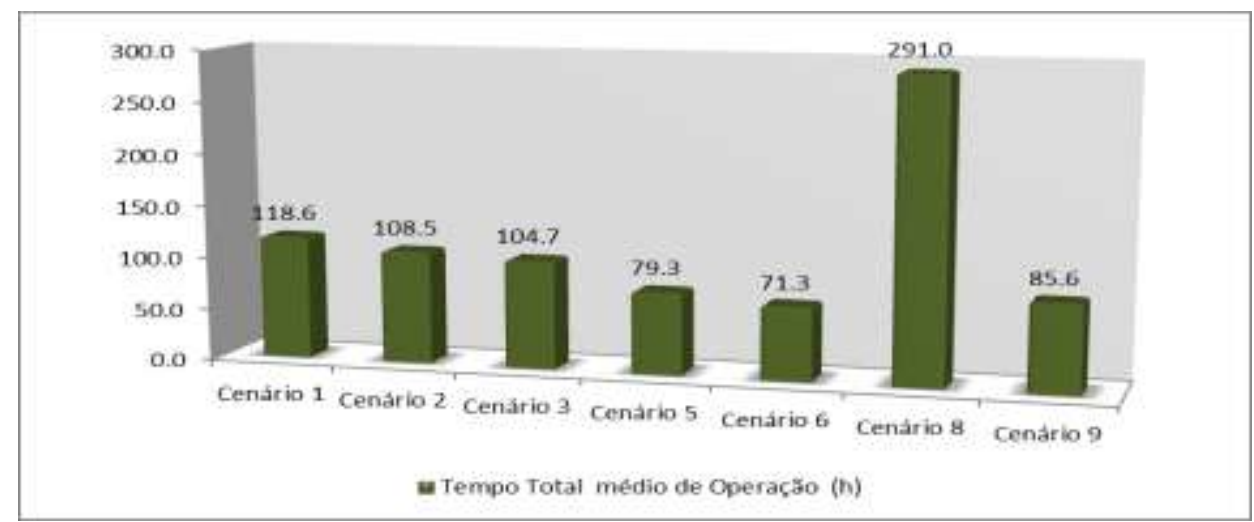

Figura 10: Tempo total médio de operação por carga (TEU)

Nota-se que o pior desempenho neste indicador é do Cenário 8, este fato é explicado pela alta taxa de ocupação deste cenário que implica em um maior tempo de espera para processamento, aumentando assim o tempo total médio por unidade de carga movimentada.

Com exceção ao Cenário 8, todos os demais cenários apresentaram melhor desempenho do que o obtido na simulação do sistema de transporte real, que obteve tempo total médio de 139.42 horas, ou seja, geram redução significativa do tempo consumido para movimentar uma capacidade carga maior do que é realizado pelo sistema real.

No que refere-se ao Tempo total médio de espera, nos diversos processos portuários, os valores expresso no gráfico da Figura 11 abaixo estão apresentados em função da unidade de carga movimentada:

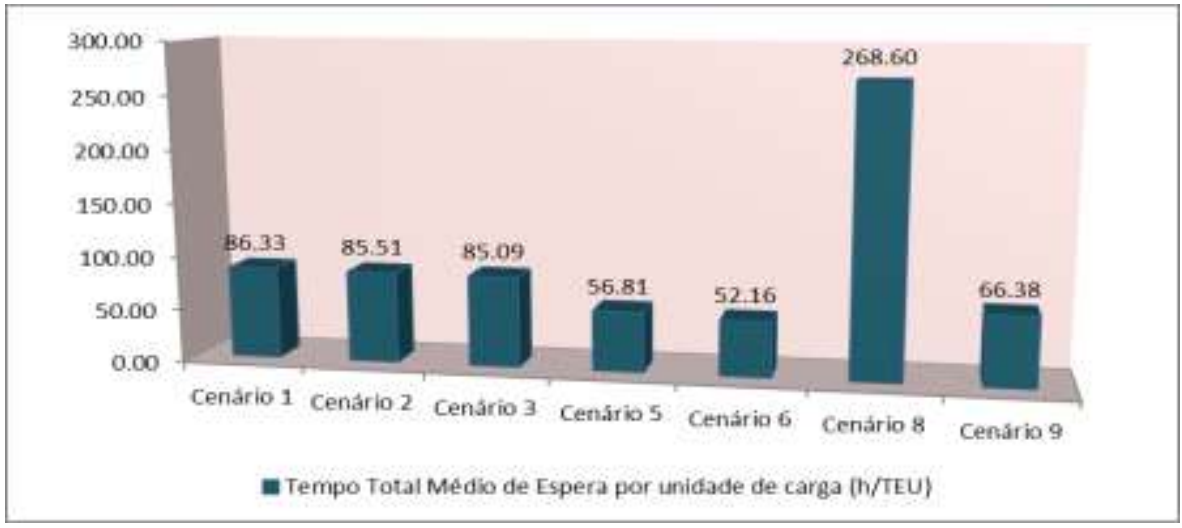

Figura 11: Tempo total médio de espera por carga (TEU)

Destaca-se os resultados obtidos pelos Cenários 5, 6 e 9. A simulação do cenário real de transporte mostrou que as cargas com origem e destino em Manaus possuem tempo de 
espera estimado em 93.2 horas, ou seja, bem acima dos resultados dos cenários alternativos, com exceção ao Cenário 8.

\section{CONSIDERAÇÕES FINAIS}

O presente estudo propôs modelos de simulação computacional para analisar o comportamento operacional da cabotagem do estado do Amazonas utilizando serviço feeder, com a adoção de um porto concentrador, para que o sistema pudesse funcionar usando a lógica de uma rede "hub-and-spoke". Para tornar possível a realização deste estudo foi adotado o uso de técnicas de modelagem e simulação que permitissem construir cenários logísticos alternativos.

Os resultados das simulações dos cenários alternativos evidenciaram que alguns cenários obtiveram resultados operacionais que permite afirmar que o serviço feeder, para determinados níveis de demanda, tornam o sistema sub-utilizado. Neste contexto, verificou-se que os cenários que apresentaram resultados mais significativos são aqueles que obtiveram o melhor equilíbrio entre demanda e capacidade operacional.

Desta forma, o porto concentrador regional é o mais indicado para atender a demanda prevista para os próximos anos (Cenário 1), não estando apto para atender maiores volumes de movimentação de conteinêres, como nos casos dos níveis de demanda potencial e super demanda.

O porto concentrador de abrangência nacional, (Cenário 5), é o mais indicado para o volume de movimentação de contêineres estimado para a demanda potencial que o estado do Amazonas pode atrair, segundo empresas do setor. Isto ocorre porque este tipo porto é subutilizado para a demanda prevista e opera saturado para uma super demanda.

O porto concentrador de abrangência internacional, (Cenário 9), apenas tem justificado seu uso no caso de uma super demanda de movimentação de contêineres por navegação de cabotagem, devido os elevados custos de implementação de um projeto deste porte. Considera-se, ainda, que para níveis inferiores de demanda o porto opera com baixas taxas de ocupação.

Estes resultados evidenciam que a viabilidade do serviço feeder, como modelo de funcionamento para cabotagem do estado do Amazonas, depende do volume que se pretende atingir na movimentação de conteinêres, bem como da disponibilidade de recursos financeiros para a construção e operacionalização do projeto.

\section{REFERÊNCIAS BIBLIOGRÁFICAS}

[1] Agência Nacional de Transportes Aquaviários. Comparativo dos Preços Médios Totais: Contêineres - 2008 e 2009: Tabela 29. Brasília, 2010.

[2] Agência Nacional de Transportes Aquaviários. Desempenho Portuário: Sistema Permanente para o Acompanhamento dos Preços e Desempenho Operacional dos Serviços Portuários On-line. Brasília, 2012. Disponível em: <http://www.antaq.gov.br/Portal/DesempenhoPortuario/Index.asp>, acesso em: 25/04/2012.

[3] Agência Nacional de Transportes Aquaviários. Marinha Mercante Brasileira. Rio de Janeiro, 2008.

[4] Banks, j.; Carson, j.s.; Nelson b.l.; Nicol d.m. Discrete-event System Simulation. 5 ed. New Jersey: Prentice-Hall, Inc., 2009. 
[5] Botter, R.C.; Medina, A. C.; Moura, D.A.; Lobo, G.; Garber, M.F.; Nosralla, G.B. Análise da situação atual de regulamentação da cabotagem no Brasil e uma breve análise da cabotagem no mundo. In: SOBENA, 2006, Rio de Janeiro. Anais. Rio de Janeiro: SOBENA, 2006.

[6] Cámara Interamericana de Transportes. Livro Transporte vol II. Transporte aquaviário (TAC). 2003.

[7] Cuoco, M. Otimização da seleção e alocação de cargas em navios de contêineres. 2008. 116f. Dissertação (Mestrado Em Engenharia de Sistemas Logísticos) - Escola Politécnica da Universidade de São Paulo, Departamento de Engenharia de Transportes, São Paulo, 2008.

[8] Medau, J. C. Análise de capacidade do lado aéreo de aeroporto baseada em simulação computacional: aplicação ao aeroporto de São Paulo - Congonhas. 2011. 123p. Dissertação (Mestradoem Engenharia de Transportes) - Programa de Pós-Graduação em Engenharia de Transportes - Escola Politécnica da Universidade de São Paulo, Departamento de Engenharia de Transportes, São Paulo, 2011.

[9] Medeiros, R. L. Análise dos serviços de cabotagem e longo curso do sistema de transporte aquaviário da cidade de Manaus. 2012. 82f. Monografia (Especialização em Engenharia da Produção) - Universidade do Estado do Amazonas - Escola Superior de Tecnologia, Manaus, 2012.

[10] Nakamura, C. Y. Análise da viabilidade da utilização do transporte por cabotagem para a movimentação de automóveis novos no Brasil: um estudo de caso. 2010. 107f. Dissertação (Mestrado em Ciências) - Escola Superior de Agricultura "Luiz de Queiroz", Piracicaba, 2010.

[11] Santos, J. T. A. N. dos; Cardoso, P.; Moita, M. H. V. Análise dos principais fluxos de cargas do sistema aquaviário da cidade de Manaus. VI Simpósio amazonense de engenharia de produção. Gestão de Operações e Serviços: Inovações e Oportunidades. Manaus, 2011.

[12] Santos, J. T. A. N. dos; Cardoso, P.; Moita, M. H. V. As paralizações na operação de embarque e desembarque de carga influenciam na produtividade das operações de cabotagem? Um estudo de caso no terminal de uso misto privativo de Manaus. MIEPEX: Manaus, 2010.

[13] Silva, M. R.; Cunha, C. B. Configuração de redes do tipo hub-and-spoke para o transporte rodoviário de carga parcelada no Brasil utilizando algoritmos genéticos. Revista Transportes, volume XI, n 2. 2004.

[14] Sistema de Informação da Navegação Interior. Relatório Estatística Portuária: Apresentação da movimentação geral de contêineres, serviços portuários e a análise estatística dos indicadores operacionais dos Portos e Terminais de Manaus/AM. Manaus: INTRA, 2012. 